Transmission curves are given for each glass on a separate chart. This supersedes the previous method of plotting densities and is in conformity with other makers' lists. The integrated visible transmission, for a standard lamp and thickness of filter, is also supplied, and a formula given by which the transmission can be calculated for any other thickness. It is believed that there are only two other makers of coloured glass of optical quality in the world, and the English list compares favourably in range with any other.

The makers are to be congratulated on the publication of the new catalogue under present conditions.

H. W. LEE.

\section{MOTION AND FORMATION OF THE PLANETS}

$\mathrm{T}$ WO papers under the title "Gravitational Theory of Planetary Rotation", and "A New Theory of Planetary Formation", by J. Miller, have just appeared (J. Brit. Astro. Assoc., 52, 5 and 6, June and July 1942). Miller shows that a spherical body or a loose agglomeration of particles, held together by gravitational force or cohesion, which is describing an orbit around a central body, will acquire a rotation in a sense opposite to that of revolution. This rotation is produced by the unequal attractions of the central body or centre of force on the near and remote sides of the smaller body, and the axis of rotation will be perpendicular to the orbital plane. The linear velocity of rotation will vary as the diameter of the body, and will also be a function of the distance between the body and the central force. Owing to the fact that a second order term appears in certain computations, the linear velocity will not be exactly proportional to the diameter, and in addition, internal friction between the different strata has an important effect.

A relation between increments of time in rotation rates and increments in diameters is deduced by Miller, and the theoretical times of rotation for the different planets are computed. Some of these agree well with the actual times of rotation, and, where discrepancies occur, it is suggested that the internal frictions of the planets, for which it is difficult to calculate exact figures, are responsible. It is assumed that the solar system once revolved around the centre of a star cluster, which, in turn, was revolving around the centre of the galaxy, and planetary rotations originated as a result of the difference in the attractions on the near and remote sides of the planets, to which reference has already been made. These rotations have been maintained since they were established, though, as pointed out, there were probably other disturbing factors which modified the original rates to a small extent.

No explanation is given in the first paper of planetary formation, but this problem is attacked in the second paper. The sun alone in the solar system is first dealt with, and it is conceived as originally describing an orbit around a centre of force which may be, as in the previous case, a stellar cluster or the galaxy. Under favourable conditions, such as approach to the periphery of the cluster, the disruptive force of the latter, due to its differential attraction, will at a certain stage overcome the combined forces of the sun's gravitational pull on its external layers and of the cohesion of the particles. A portion of the sun will be removed, and as it makes closer approaches to the cluster, more material will be torn off. Miller suggests that the outer planets were removed first, and as the disrupted sun moved closer to the cluster, it lost more and more material which formed the remaining planets, Pluto being the first to be removed at a great distance and Mercury the last when the sun was nearest to the centre of attraction.

The theory differs from the planetesimal hypothesis of Chamberlin and Moulton in many ways. A cluster, not another star, is held to be responsible for the disruption. In addition, internal forces in the sun, caused by the high temperature, are not considered necessary to assist with the ejection of matter. A tentative explanation of Bode's Law is given, but this is not worked out in detail. The physical condition of the sun at the time of the formation of the planets is left an open question; it may have been in a state somewhat resembling its present condition, or it may have been in a seminebulous state. It is assumed that the matter composing the superficial layers did not all possess the same cohesive force, from which it may be inferred that the sun was not entirely nebulous at the time of the disruption, though it may have been very much more extended than it is at present. Miller's theory requires that the outer planets, which were first disrupted, should possess less cohesion than those closer to the sun, so that the cohesive power should increase from Mercury outwards. As, however, great changes have taken place in the planets since their formation. this rule cannot be applied very strictly.

\section{INDIAN FORESTRY RESEARCH*}

$\Delta \mathrm{S}$ a record of useful economic activities, a report A entitled "Forest Research in India and Burma, $1940-41$ " is an encouraging summary of many-sided investigations under difficult conditions. It deals with the work of the Forest Research Institute at Dehra Dun during the period under review. Naturally the outstanding feature of the year's activities has been the influence of the War. At first the demands made upon the staff of the Institute were connected with supplies, but as its work is primarily research it was for this reason capable of dealing only with comparatively small quantities of materials. It became, therefore, wisely agreed that the Institute could not make its best war contribution by turning itself into a supply unit. It has confined its programme in consequence to research work in connexion with various war problems.

While the activities of the Utilisation Branch of the Research Institute were largely engaged upon war work, rather than normal programmes, those of the Botanical and Sylvicultural Branches were more especially engaged on the normal research and other duties where interruption would lead to waste of previous efforts. In the Utilisation Branch many inquiries were dealt with in connexion with timbers for various war uses. These included bamboos for aircraft work and tent poles; timbers for rifle parts and tool handles; containers for ammunition, for packing army boots, etc. As an example it may be

* Forest Research in India and Burma, 1940-41. Part 1: The Forest Research Institute. Pp. 161. (Manager of Publications, Delhi, 1941.) Rs. 1.12 or 28 . $6 d$. 